\title{
A QoS Monitoring System in a Heterogeneous Multi-Domain DVB-H Platform
}

\author{
Hamidreza Shirazi, Member, IEEE, John Cosmas, Senior Member, IEEE, Thomas J. Owens, \\ Yong-Hua Song, Senior Member, IEEE, and Angelo Centonza, Member, IEEE
}

\begin{abstract}
The MobileTV, IPTV, and DVB standards (DVB-H/T) have been defined to offer mobile users interactive multimedia services with quality of service $(\mathrm{QoS})$ consistency analogous to $\mathrm{TV}$ services. However, the market has yet to provide effective and economical solutions for the real-time delivery of such services to the corresponding transmitters over multi-domain IP networks. The monitoring system proposed in this paper enables the QoS in the IP networks involved in the delivery of real-time multimedia content to the transmitters to be ascertained. The system utilizes the QoS parameters defined in MPEG-2 Transport Streams to detect problems occurring in the heterogeneous multi-domain IP networks. The ability to detect problems having an adverse effect on QoS allows appropriate control actions to be determined to recover the QoS across the composite IP network. The design and implementation of the proposed QoS-Monitoring system (QoS-MS) is presented, followed by analysis of experimental results that demonstrate the feasibility of the system.
\end{abstract}

Index Terms-DVB-H, DVB-T, IPTV, QoS Monitoring, SNMP.

\section{INTRODUCTION}

$\mathbf{T}$ HE vision of cooperation between MPEG-2 broadcasting and IP-based mobile data communication networks is rapidly becoming a reality [3], [4]. Many commercial mobile TV networks are likely to be hybrid networks combining a uni-directional broadcast network with a bi-directional mobile telecommunications network. A terrestrial TV broadcast network transmitter typically offers a wide transmission area. However, a mobile broadcast network transmitter typically covers a much smaller transmission area (a cell). A technology such as Digital Video Broadcasting-Handheld (DVB-H) is well suited for delivering high quality broadcast services from various sources to large numbers of mobile receivers [11]. However, it is necessary to employ ubiquitous backbone networks covering large geographical areas to deliver multimedia services to remote DVB-H gateways in cellular DVB-H networks. Such backbones are likely to be IP based due to the availability of the Internet worldwide. Hence, in an environment of multi-domain convergent networks one of

\footnotetext{
Manuscript received October 30, 2007; revised October 14, 2008. Current version published February 25, 2009. This work was supported in part by EU under the IST program as the project INSTINCT (IP-based Networks, Services and Terminals for Converging systems), IST2003-507014.

H. Shirazi, J. Cosmas, and T. J. Owens are with the School of Engineering and Design, Brunel University, Uxbridge UB8 3PH, U.K. (e-mail: hamidreza.shirazi@brunel.ac.uk; john.cosmas@brunel.ac.uk; thomas.owens@brunel.ac.uk).

Y.-H. Song is with Liverpool University, Liverpool L69 3BX, U.K. (e-mail: hua.song@liv.ac.uk).

A. Centonza is with Roke Manor Research Limited Romsey, Hampshire, SO51 0ZN, U.K. (e-mail: angelo.centonza@ roke.co.uk).

Color versions of one or more of the figures in this paper are available online at http://ieeexplore.ieee.org.

Digital Object Identifier 10.1109/TBC.2008.2010371
}

the biggest challenges is to deliver real-time DVB-H services with a quality of service (QoS) acceptable to the end-users. Addressing this challenge is considered crucial to the success of such convergent services [6].

Apart from the "lossy" nature of the IP networks feeding the DVB-H transmitters, there are many other factors that are detrimental to the delivery of satisfactory QoS to the end-users. These include wrong configuration of the network resources, Service Level Agreements (SLA) and/or Service Level Specifications (SLS), and the DVB-H gateways. Moreover, IP network congestion, packet loss, packet delay, and delay jitter, especially in the case of real-time video transmissions, can cause service interruption or performance degradation.

The above issues could represent a threat to the future of DVB-H services as most IP networks are still best effort. Moreover, problems with QoS guarantees can arise in IP domains using Diffserv techniques due to the limited number of service classes, the presence of "greedy applications", and the lack of guaranteed end-to-end QoS [14]. The situation can be even worse when the sender and the receiver of the service contents are far apart. This situation implies the presence of a number of IP domains along the service route resulting in a higher probability of network congestion and performance degradation [3], [4], [14].

There have been various activities aimed at providing a reliable and scalable platform for delivering services with a QoS defined in service agreements. For instance, the IST project INTERMON [20] came up with a solution to guarantee QoS at the inter-domain level but it suffers from scalability issue that is likely to pose problems in the Next Generation Networks (NGN). The IST project MoMe [21] focused on inter-domain QoS monitoring and analysis proposing a real-time QoS architecture with integrated monitoring and measurement capabilities. The IST project SCAMPI [22] focused on QoS monitoring in the Internet using passive monitoring adapters and other measurement tools to tackle the Denial of Service (DoS) issue, SLS auditing, traffic engineering and traffic analysis, etc. The IST project LOBSTER [23] advanced the European Internet Traffic Monitoring Infrastructure using a similar method to that was suggested in the IST project SCAMPI. The IST project AQUILA [24] proposed an inter-domain QoS metrics measurement mechanism using the Border Gateway Resource Protocol (BGRP) to support measurement based Admission control (MBAC) in IP-based networks. The IST project ETHRONE [25] came up with an inter-intra domain end-to-end solution for monitor the audio/visual streams in IP-based heterogeneous platforms. It employed service level monitoring components communicating in a cascaded form and measurement information translated into a network independent format (i.e. XML). The monitoring components were deployed across the platform and right at the customer level to report the customer perceived 
QoS to a monitoring center which assessed the data reported based on the measured network performance. The solutions proposed for resolving detected issues were based on short to long term decisions on optimizing network usage and resources as well as cross layer adaptation [26]. These solutions might be viable in the ETHRONE context as the project considered a business model involving content providers, service providers, network providers, content consumers. However, the convergence of telecommunication systems and broadcasting systems will most likely lead to close cooperation among the parties involved (service providers, network providers, etc.) rather than a full convergence of operators. Therefore, a central monitoring center responsible for monitoring and control the end-to-end QoS across the whole platforms can't be a practical solution as parties involved in creation and distribution of services (i.e. content providers, network providers, etc.) are reluctant to expose their backbone to other parties. In addition, the network convergence is rapidly becoming a reality and delivery of contents using the best-effort IP backbone is now more common. Hence, a single operator like a broadcast operator is facing a new challenge to monitor and control QoS in a multi-domain network reliant on a wide range of network providers.

This paper proposes a practical solution to enable a broadcast operator to actively monitor and possibly control the QoS across the heterogeneous platforms. The control function, which is briefly studied herein, is proposed as a quick response to the QoS degradation to retain the customers' satisfaction. As a broadcast network is typically MPEG-2 based, a QoS monitoring system is needed to enable the QoS throughout the multi-domain contribution network to be accurately estimated by a broadcast operator that can only actively monitor services on MPEG-2 traffic routes. The system has to allow the broadcaster to detect the degradation of QoS, distinguish and analyze the root-cause(s) of the degradation, and apply appropriate on-the-fly control action(s) to recover the QoS across the composite IP network.

The state-of-the-art QoS-Monitoring System (QoS-MS) proposed herein is different from previous proposals in that it is based on a fact that a single broadcast operator does neither have permission for inter-domain network engineering nor intention to actively monitor the IP-based networks. However, the QoS-MS allows the broadcast operator to deduce the overall status of the multimedia delivery network based upon MPEG-2 measurements reported from broadcasting edge (i.e. DVB-H gateways). The QoS-MS is a viable and cost-effective solution utilizing MPEG-2 measurements and Simple Network Management Protocol (SNMP). The reporting system employed in the QoS-MS uses the HTML and XML formatting to present monitoring data to the operator to locate and identify the cause of the degradation detected by the monitoring probes deployed at the DVB-H gateways. The proposed system is aimed to provide the broadcast operator with succinct monitoring information and connectivity features to deliver TV-like services to mobile devices over IP-based and DVB-H networks.

The paper is organized as follows. In Section II, the QoSMonitoring System (QoS-MS) is introduced and the design of the system is described. In Section III, the measurement parameters used, the test-bed set-up and the experiments carried out

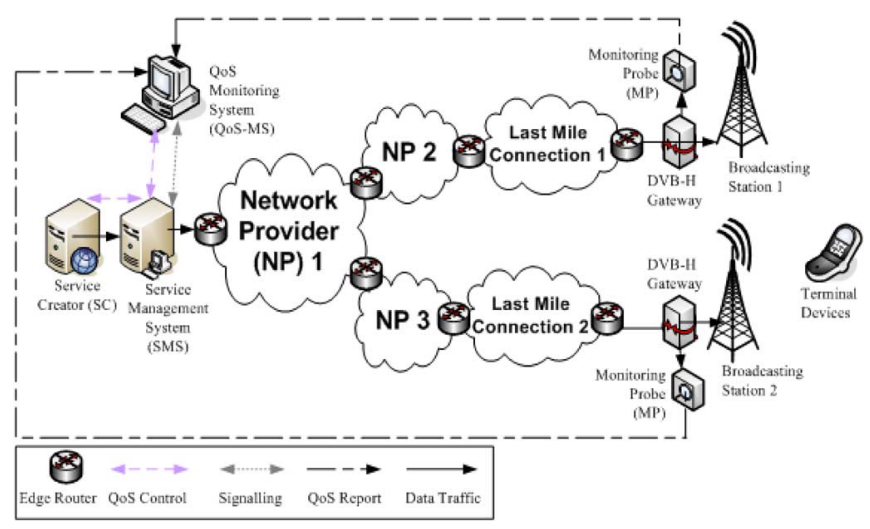

Fig. 1. Signaling, reporting, and actions in the QoS-MS.

are described. The performance of the QoS-MS in the emulated environment is evaluated on the basis of the experimental results obtained. Finally, the conclusions are given in Section IV.

\section{QoS-MONITORING SYSTEM}

\section{A. Concept}

There have been various standards and ratifications for network monitoring and management. The International Organization for Standardization (ISO) addressed five major functional areas of the Network Management Model as performance management, configuration management, security management, accounting management and fault management [13]. The Network Resource Architecture (NRA) proposed by Telecommunications Information Networking (TINA) provides a generic information model of network resources hosting multimedia services [1]. In addition, the Distributed Management Task Force (DMTF) has ratified standards in Management Initiatives, Common Information Model (CIM), Web-based Enterprise Management (WBEM) and Desktop Management Interface (DMI) [8]. DMI provides a standard framework for managing and tracking components remotely and exchanging management information securely [9], [10]. This paper utilizes results of [5] and related standards to propose a QoS-MS system for real-time multimedia services delivered over IP networks to a broadcast network. The QoS-MS system is viable for existing DVB-H network operators enabling the broadcaster to monitor and control the quality of real-time multimedia services delivered to its DVB-H gateways over IP based networks.

Fig. 1 shows the block diagram of the multi-domain infrastructure. In this architecture, the Service Creator (SC) receives contents from different sources, like satellite links or video cameras, and re-encodes them in a format suitable for DVB-H transmission. The contents are delivered to the Service Management Subsystem where the services are aggregated and sent to the DVB-H gateways over a multi-domain IP-based network. Once the services reach the gateways, they are encapsulated in MPEG-2 packets and delivered to the broadcasting transmission towers. The QoS-MS system collects monitoring and management data from various sites of the network. This data is processed to provide the broadcast network operator with useful reports about the state of the network. Consequently, the operator is able to ascertain the performance of the network, for 
instance, by checking the service availability, service encoding and traffic re-encoding. If performance degradation is detected along the delivery network, the operator is told by the QoS-MS to take appropriate action. One of the actions might be to reduce the traffic bit rate at the service encoder/play-out to relieve congestion commonly encountered in IP domains [4].

The QoS-MS assists the operator in quickly diagnosing erroneous behavior in network domains involved in the delivery of service contents. Consequently, this can result in improving the post-sale services by shortening the mean time to repair (MTTR) and the mean time between failure (MTBF); hence improving the customers' satisfactory level and ultimately preventing potential loss of revenue.

\section{B. Design}

The design of the QoS-MS is characterized by object oriented, distributed and loosely coupled software components and follows the separation of concern principle. It conforms to the TINA-C reference model [1] and covers the classical ISO management categories. It adapts the Umbrella Management concept [15] and utilizes the Simple Network Management Protocol (SNMP) to achieve cross domain management. The QoS-MS is based on a 3 level architecture:

1) The Remote Management level: contains the Monitoring Application which manages the Probe Agent remotely using SNMP protocol over the composite IP network. The remote application is web-based and provides real-time graphical views of the network status.

2) The Agent level: the "Probe Agent" is an intermediary between the QoS-MP and the remote application which reports the QoS measurements using SNMP-Management Information Base (MIB) knowledge [15].

3) The Probe or Instrumentation level: contains the instrument called "QoS Monitoring Probe (QoS-MP)" and is located along the MPEG-2 Transport Stream (TS) service route. It measures and records the MPEG-2 QoS parameters.

The QoS-MP contains the Probe Agent and MPEG-2 Analyzer. The Probe Agent is an interface between the remote Monitoring Application and the probe. It evaluates the MPEG-2 measurements and sends/receives monitoring-related information to/from the Monitoring Application. The SNMP protocol is implemented in the functional elements of the QoS-MS including the QoS-MPs and Monitoring Application. A common SNMP-MIB database needs to be registered and deployed in the QoS-MS. This SNMP-MIB database uses a tree structure for presenting data and contains the parameters defined for MPEG-2 QoS analysis. These parameters can be the Transport Stream (TS) Synchronization, Cyclical Redundancy Check (CRC), Program Clock Reference (PCR), Presentation Time Stamps (PTS) Repetition, PCR Jitter, Program ID (PID), and Video and Audio Bit rate [12]. The Probe Agent compares the results of the MPEG-2 measurements with the corresponding thresholds pre-set for each parameter by the broadcast operator. It generates an alert (alarm) when a parameter exceeds or fails a threshold. The alerts are gathered in monitoring reports and sent to the

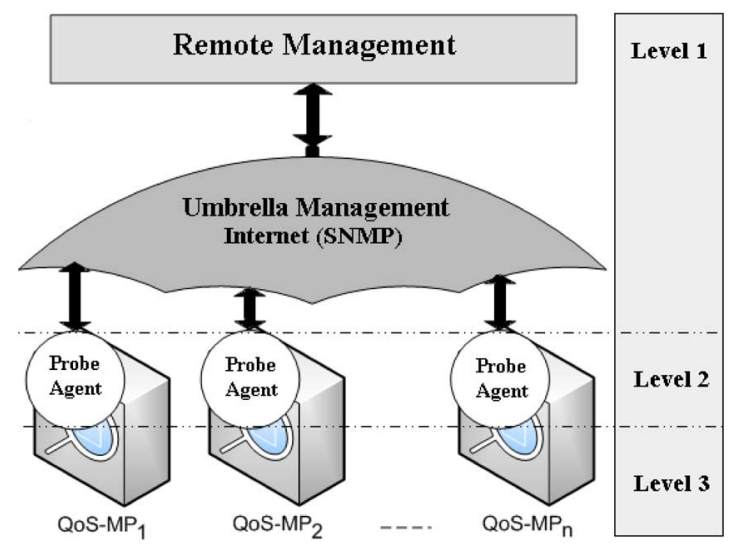

Fig. 2. The 3-level QoS-MS architecture model.

remote server in SNMP Trap Protocol Data Units (PDU) [15]. The reports state the broadcast service traffic monitored and indicate the state of the services delivered from the play-out to the broadcasting towers. The main duty of the Probe Agent is to keep the Remote Management informed of the network status.

The Remote Management level of the system consists of the remote Monitoring Application that offers network monitoring and control features to the broadcast network operator. The Monitoring Application incorporates the objects: Main Application, SNMP manager, Database adapter, Report generator and Web-based interface. The Main Application manages the performance of the Monitoring Application by assigning specific tasks to each object. The SNMP manager enables the operator to configure and control the Monitoring Probe(s) using SNMP-MIB variables. The SNMP Manager performs the SNMP related tasks such as establishing SNMP connections, sending SNMP commands and/or receiving SNMP traps. It processes the SNMP PDUs and provides the Main Application with the monitoring data extracted from monitoring reports of the SNMP traps. The Main Application passes the monitoring data to the Database adapter to be saved in an appropriate format in the database. The Database adapter deals with the database transactions including add/remove records and running queries on the database. The Report generator puts the monitoring data into a web-based format (i.e. XML file) and feeds it to the Web-based interface which presents it graphically in HTML format. The Web-based interface enables the operator to submit a request, either for monitoring or control purposes, to the network at any time. Depending on the nature of the request, the Web based interface, either passes it to the Report Generator or the SNMP Manager.Fig. 2 presents a graphical view of the 3-level system architecture of the QoS-MS.

The MIB variables are initialized across the network using SNMP manager integrated in the Monitoring Application based upon the contracted SLAs. The parameters of the SLAs can be enforced either manually by the broadcast network operator or automatically through the Service Management Subsystem. The Service Management Subsystem can send an XML file containing the corresponding values of the SNMP-MIB variables to the Monitoring Application. The Monitoring Application also needs to be notified of the service encoding parameters applied by the SC. The service encoding parameters 
are used to interpret errors due to performance degradation in the multi-domain backbone network and validate whether the encoding parameters set in the SC are in accordance with the SLAs contracted. These parameters are provided to the Monitoring Application by the SC through the Service Management Subsystem which directly interacts with the SC for monitoring and control purposes. The SNMP-MIB variables and service encoding parameters are defined based on [18] and may include parameters like TS Synchronization, Sync Byte, Program Association Table (PAT), Continuity Count, Program Map Table (PMT), Program ID Distance, Transport, Cyclic Redundancy Check (CRC), Program Clock Reference (PCR) Discontinuity, PCR Repetition, PCR Jitter, Presentation Time Stamps (PTS) Repetition, TS/Video/Audio Bit Rate and Single Frequency Network (SFN) Synchronization.

In the heterogeneous network (see Fig. 1), the Service Management Subsystem is updated about the network status and level of QoS measured at each QoS-MP. Therefore, when an error is detected the Service Management Subsystem can promptly identify the problem and take action to re-establish satisfactory end-to-end QoS. The remote Monitoring Application can be programmed to fix the problem automatically or as a result of operator input. The design and implementation of Service Management Subsystem and SC [5] are out of the scope of this paper.

Fig. 3 presents the component diagram of the QoS-MS wherein its functional components and their interactions inside and outside of the system are shown.

The monitoring reports are sent from the Monitoring Probes to the Monitoring Application. They convey monitoring data to enable the operator to locate problems and discover sources of error in the network. Hence, the monitoring data should at least include the IP address of the reporting Probe, date, time, type (alarm, information, etc.), state (new, continued), a short description of the event together with its associated value, and the relevant program ID. The Monitoring Application is prompted upon receiving monitoring reports to process and present the measurement reports in a web-based format.

The next section describes the test-bed used to validate the QoS-MS concept and analyzes the performance of the QoS-MS system in a laboratory environment.

\section{TEST-BED EXPERIMENTS AND RESUlt ANALYSIS}

\section{A. Measurement Parameters}

One of the novelties of the QoS-MS is the use of standard MPEG-2 compliant measurements. Although these measurements are collected on MPEG-2 service routes, they are employed for detecting anomalous behavior in IP domains taking part in the broadcast content delivery. The measurements used for this purpose were mainly of the following three types:

1) "MPEG-2 Audio and Video Bit Rate" measurements provide important information about the service bit rate in the multi-domain IP network. If the recorded bit rate is lower than expected and no MPEG-2 Packet Continuity Count errors are monitored [12], it can be concluded that the backbone multi-domain IP network is not providing enough

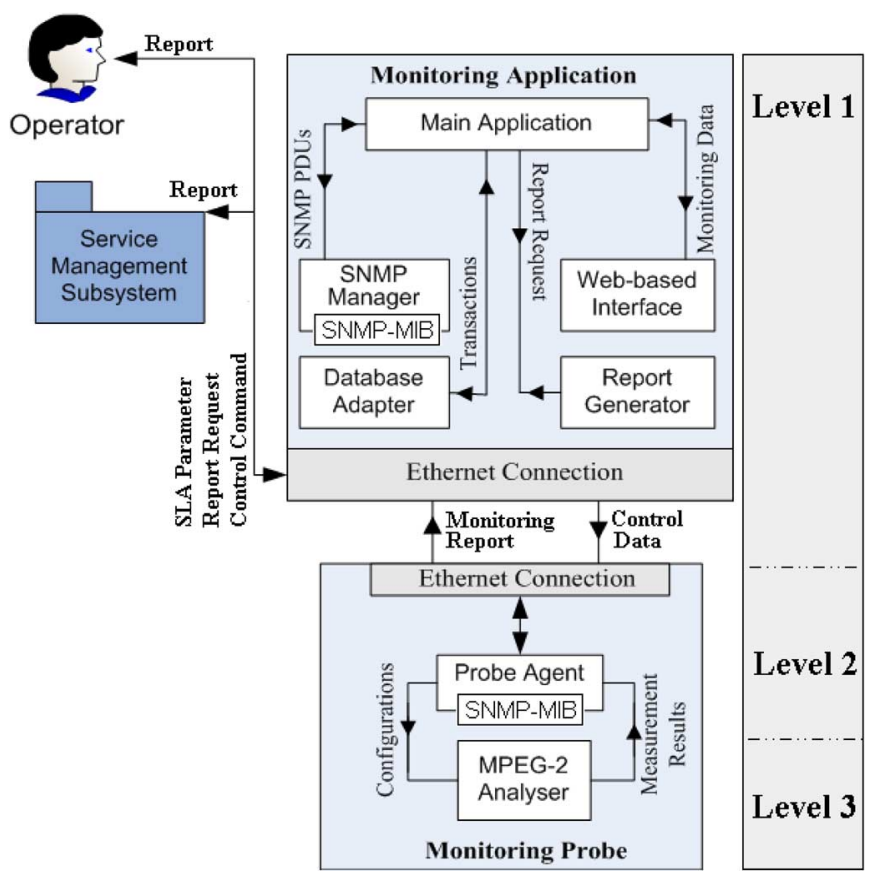

Fig. 3. Component diagram of the QoS-MS.

bandwidth for the effective delivery of the monitored services.

2) "PID Error" measurement provides information on whether traffic streams related to different service contents reach the DVB-H gateways. If such an error is monitored it means that the backbone multi-domain IP network has experienced a performance degradation of such a magnitude that it has resulted in an interruption of data transfers.

3) "Null Packets Bit Rate" measurements can be used to detect events similar to those derived from the audio and video bit rate monitoring. In fact, the bit rate set up in a MPEG-2 multiplexer channel is always higher than the overall bit rate consumed by the service contents so as to allow provisioning of sufficient bandwidth. Therefore, part of the channel bandwidth is used for transmission of null packets. The average value of this bandwidth is normally constant. If the average value of this bandwidth increases and no errors related to the MPEG- 2 streams are monitored, it means that the bit rate available to some of the content streams has decreased. Consequently, it can be deduced that the multi-domain backbone IP network is not providing the bandwidth needed for the correct transmission of all the services.

\section{B. Test-Bed Set-Up}

The effectiveness of the proposed QoS-MS was tested in a laboratory environment simulating the behavior of real multidomain IP networks during the real-time delivery of TV-like services to DVB-H/T gateways. The reason for the choice of this type of service is that DVB-H was primarily standardized for the broadcast of TV-like data and content to hand-held devices like mobile phones.

In the test-bed, a video camera was used to capture live video sequences and generate a PAL (Phase Alternation 


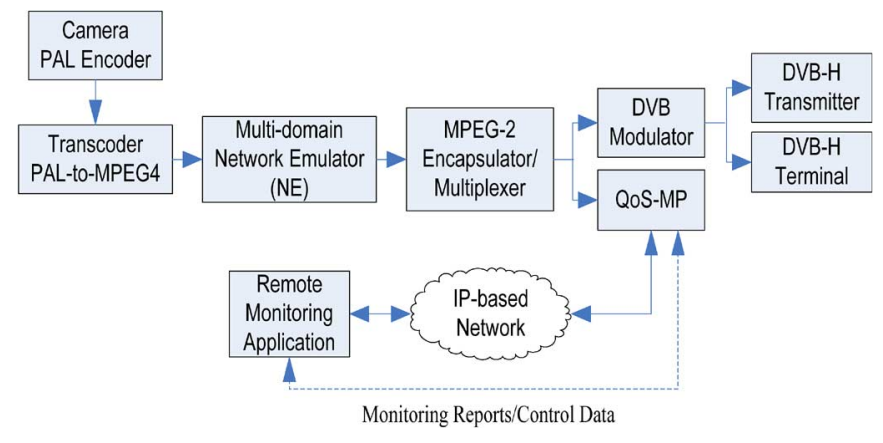

Fig. 4. The laboratory test-bed for evaluating the QoS-MS.

by Line) signal. The Service Creator (SC) is a passive Audio/Video Transcoder/Transrater that was connected to the video camera to generate an MPEG-4 stream at a bit rate of 256kbps. The output stream of the SC was fed to the Network Emulator (NETEM) [19] as IP traffic. The NETEM was a Linux Debian based network emulator [7] used to reproduce the behavior of a real multi-domain contribution network and in particular resemble the case of service resource denial on the backbone network by setting network performance parameters such as Bit rate, Packet loss and etc. The NETEM was used to simulate the composite IP network by controlling the packet loss percentages, packet delay, delay jitter, and available service bandwidth. The emulated signal was then introduced to the DVB-H Gateway. The DVB-H Gateway is an integrated DVB-H MPEG-2 Encapsulator and Multiplexer which provides an MPEG-2 Transport Stream (TS) output compliant with the time slicing techniques adopted in the DVB-H standard. The MPEG-2 TS was fed to the QoS-Monitoring Probe (MP) and DVB Modulator. The QoS-MP was an MPEG-2/IP analyzer performing extensive analysis of MPEG-2 transport streams and de-capsulated IP streams based on the measurement parameters. It was integrated with the Probe Agent which reported the measurement results of the analysis to the remote Monitoring Application via SNMP protocol. The Monitoring Application is a remote network management sub-system running on a computer desktop platform. It collects the SNMP-Trap PDUs from the Probe Agents and alerts the operator if anomalous events are detected. The SNMP-MIB database was built by the broadcast operator using the SNMP manager interface. The SLAs parameters and service encoding parameters were all set manually in a database used by the Monitoring Application. After modulating the MPEG-2 TS based on the DVB-H standard, the signal was delivered to a DVB-H terminal. The DVB-H terminal decoded the MPEG-2 TS in accordance with the DVB-H specification. The streaming service was played on the DVB-H terminal for post-processing.

Fig. 4 shows the test-bed developed to evaluate the performance of the QoS-MS in monitoring and control the QoS of the DVB-H delivery platform.

\section{Test-Bed Experiments and Evaluation}

The experiments performed consisted of two phases: in the first phase the NETEM was set in such a way as to allow a maximum traffic of $400 \mathrm{kbps}$ with negligible packet losses following a normal distribution with a mean of $0.1 \%$. The SC provided to

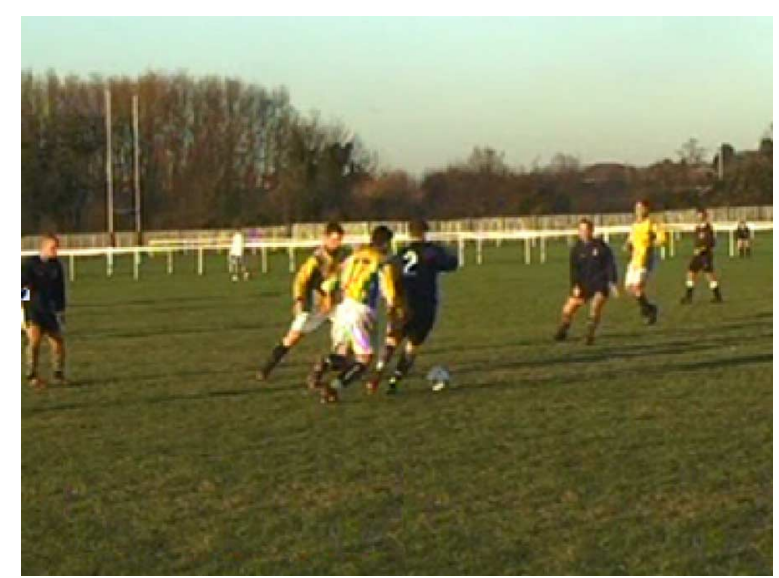

Fig. 5. Picture quality when the video stream is encoded at $256 \mathrm{kbps}$ and no bandwidth restrictions are emulated.

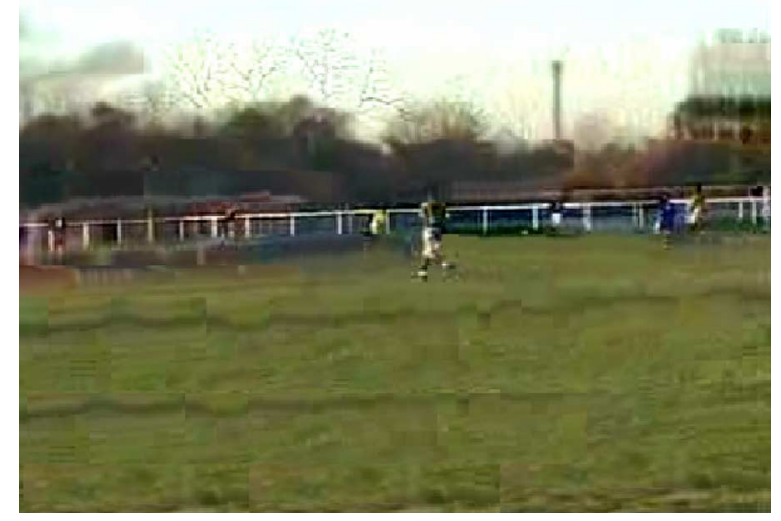

Fig. 6. Picture quality when the video stream is encoded at $256 \mathrm{kbps}$ and service bandwidth available is $200 \mathrm{kbps}$.

the NETEM an audio/video streaming service, which was encoded at $256 \mathrm{kbps}$ consisting of the traffic stream captured by a video camera. The streaming audio/video service does not encounter any bandwidth restriction and although it is affected by losses it can still guarantee a good quality of picture, see Fig. 5.

In the second phase of the experiments the NETEM was set so as to let through traffic limited to $200 \mathrm{kbps}$, while the packet losses were left unchanged. This event corresponds to an unexpected service bandwidth drop imposed by the IP network provider(s) in a real network scenario. The effect of the network performance led to service interruption and degradation of the picture quality, as shown in Fig. 6.

Under the above conditions the QoS-MP started monitoring an "Audio \& Video Bit Rate Lower than Expected" event, while no measurements affecting the quality of the MPEG-2 TS were recorded. Also, a "Null Packet Bit Rate Higher than Expected" was monitored due to the increase in the unutilized bandwidth in the multiplex channel. The IP Address of the probe, date and time of the event, together with event information including the measured value and description, event class, the event status, and the PID were passed to the Remote Monitoring Application, which in turn raised an alarm. The alarm was stored in the database and reported via an XML file made accessible via the 


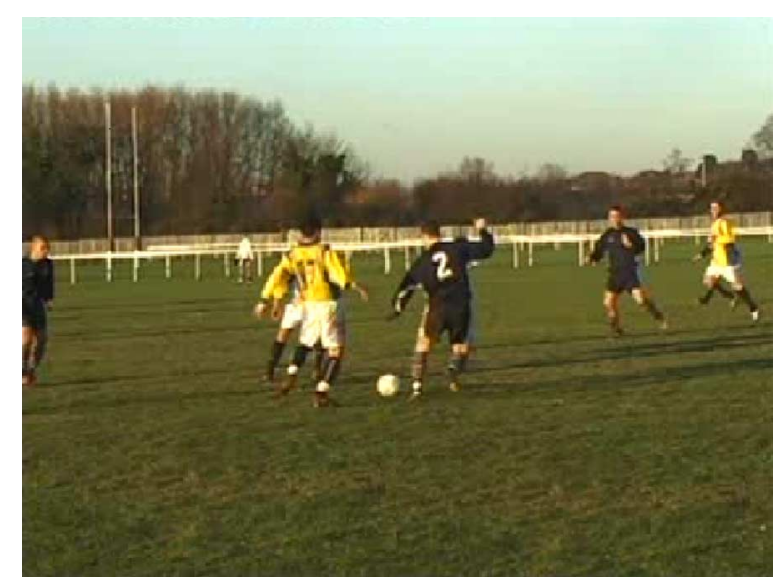

Fig. 7. Picture quality when the video stream is encoded at $192 \mathrm{kbps}$ and the service bandwidth available is $200 \mathrm{kbps}$.

Web based interface. After checking for possible erroneous encoding setups, the XML file was displayed to the operator who then reduced the encoding bit rate to $192 \mathrm{kbps}$ in the SC. The effects of this bit rate reduction on the user perceived QoS can be seen in Fig. 7, where picture quality deterioration with respect to Fig. 5 can hardly be detected.

These results should be interpreted considering that the streaming service analyzed consisted of a fast moving audio/video sequence to be presented on mobile terminals with a screen size typically of $3.5^{\prime \prime}$. Indeed, the quality of the picture when the bit rate is reduced, for all "static" video sequences and most fast moving ones, with a slight degradation for sequences with very fast camera swings, will resemble that of Fig. 7. However, such degradation in the picture quality will be preferred by the end user to service interruption or QoS degradations as shown in Fig. 7.

During the experiments, the NETEM was set to interrupt the traffic transmission from the SC to the DVB-H gateways, resembling data stream interruptions on the multi-domain backbone network. In this case, the QoS-MP started monitoring PID Errors for all the service contents affected by interruptions, while no measurements concerning degradation in the MPEG-2 transport stream were recorded. Under such circumstances the Web based report provided by the QoS-MS clearly showed the existence of a problem on the IP backbone network and alerted the operator of the event. Fig. 8 presents a sub-section of the XML report generated by the QoS-MS. The XML file describing network status is then reformatted to a user-friendly (i.e. HTML format) form with a variable refresh rate (i.e. two minutes). Fig. 9 shows the HTML time framed network report generated from XML files. The left panel of the report shows the status of each service as identified by its program ID (PID) in the network. The right panel of the report gives the details of an alarm flag in the left panel that shows errors associated with a high bit rate in PID numbered 1201 detected by the MP with an IP address of 192.168.2.16.

In the above cases, the operator recorded a mean time to repair (MTTR) of one minute based on the measurement reports received from the real-time QoS-MP. The QoS-MP categorizes the alarms so that the operator can distinguish new

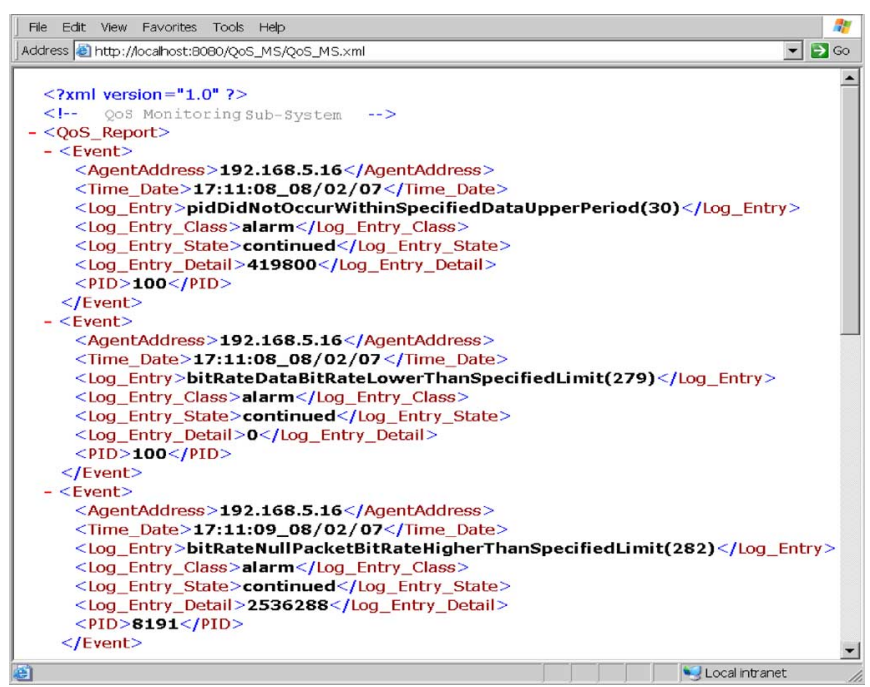

Fig. 8. An XML monitoring report generated by the QoS-MS.

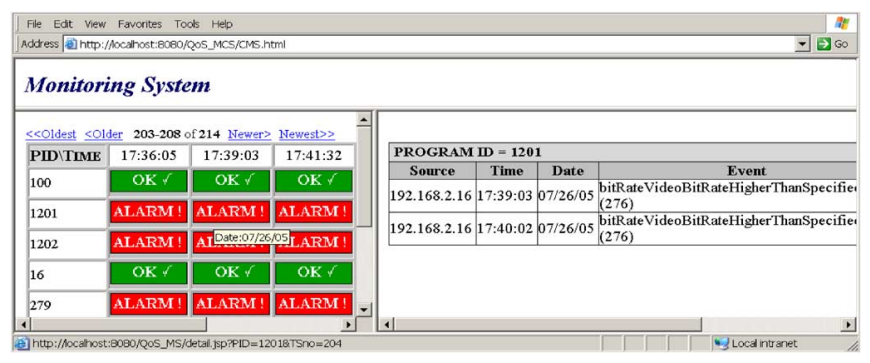

Fig. 9. The HTML times framed network report.

from continued ones. When the QoS criteria are met, the related alarms will be discontinued. Therefore, the operator can calculate the MTTR from when a specific alarm is received until it is cleared. Hence, the operator does not necessarily need to consider other variables which affect the MTTR calculation (i.e. encoding delay, transmission delay and reporting delay).

In general, the encoding and reporting delays very much depend on the implementation method, and the transmission delay depends on the network engineering and traffic status. However, in a controlled and small scope IP-based network where the transmission delay is negligible, if a Group-of-Picture (GoP)based rate control Transcoder is used, the impact of the change in bit rate on QoS will be seen in the subsequent GoP, i.e. after the current cluster of I, P and B frames has been encoded. Therefore, the transrating delay (encoding delay) will depend on the GoP duration. Typically, with a GoP of 15 frames and encoding rate of 30 frames per second, the transrating delay might reach a maximum of 0.5 seconds, and a mean of 0.25 seconds.

For delay jitter, it is worthwhile noting that if the delay jitter is within the limits of frame presentation time at the decoder, then the packet will be considered as valid and decoded as usual. If the delay jitter causes the packet to be presented at the decoder outside the acceptable presentation time (i.e. too late) then the packet will be discarded as if there was extra packet loss. In that case the FEC will try to recover the frame(s) lost. Thus, the delay jitter results in either normal decoding (for moderate jitters) or in packet loss. 
Analysis of the results presented in this section reveals how the SNMP-XML based QoS-MS effectively addresses the problem of QoS monitoring and control in heterogeneous platforms supporting DVB-H service transmission. The system allows broadcast operators to perform service monitoring only on the parts of the network directly managed by them, i.e. MPEG-2 TS routes. However, the measurements can provide useful information not only about the performance of MPEG-2 based traffic routes, but also about the IP based multi-domain backbone network supporting data transmission from remote play-outs to DVB-H gateways. Measurements collected by the QoS-MPs can be used for triggering control actions aimed at re-establishing acceptable QoS for the end users and alerting operators of unexpected events effecting QoS.

A solution such as the one proposed for QoS monitoring and control is essential to the future of DVB platforms targeting the provisioning of high quality real-time services to mobile users. The QoS-MS proposed in this paper guarantees up to standard QoS levels and efficient network management without which operators face low service penetration with serious implications for revenue. The effect of QoS on technology penetration and success is evident from the results of a fast user trial of interactive services depending on convergence and the service scenarios that benefit most from them [6].

\section{CONCLUSIONS}

In this paper a novel Quality of Service Monitoring System (QoS-MS) for DVB-H service delivery platforms is presented. The system utilizes known technologies such as MPEG-2 measurements, SNMP, XML and Java programming language to provide an original solution for QoS management and control over multi-domain IP/MPEG-2 distribution networks. The QoS-MS allows broadcast operators to monitor service contents on MPEG-2 Transport Stream (TS) routes, gathering QoS information about the multi-domain platform. This is an advantage because broadcast operators can often gain easy access to MPEG-2 streams, while they are not able to directly monitor traffic on IP backbone network domains as they are managed by external operators adopting restricted access policies. The QoS-MS enables the detection of events effecting QoS together with their location (IP or MPEG-2 domains) across the platform. It provides a Web-based XML interface for the automatic exchange of event information and the triggering of QoS control actions. It also provides a graphical Web-based interface for human operators aimed at supplying all the information needed for possible error correction and system reconfiguration.

The QoS-MS only adopts standardized DVB MPEG-2 measurements. This is a very valuable feature because the monitoring equipment existing at present for broadcasting networks implements the same standard measurements used by the QoS-MS. Hence, broadcast operators could use their existing monitoring equipment for QoS monitoring and control of IP/MPEG-2 service delivery networks by implementing SNMP-MIB features and interfacing them via the system structure proposed for the QoS-MS. In this way the broadcast network operators will be able to monitor QoS over the whole delivery platform and make sure that the services are delivered in accordance with the contracted SLAs. Therefore, the adoption of the proposed QoS-MS architecture combined with the use of already deployed measurement tools would allow reductions in the cost of system infrastructure upgrades for QoS monitoring and control with maximum accuracy.

The QoS-MS proposed in this paper could provide a benchmark for future QoS monitoring and control techniques in multidomain DVB networks. Indeed, it may be the right solution in a truly converged telecommunications and broadcasting platform where several operators take part in end-to-end service delivery [16].

It is possible to incorporate into the QoS-MS an automated subjective quality measure. The INSTINCT project partner Rohde \& Schwarz has a tool that measures the perceived quality of a DVB-H stream. Therefore, it is possible to feed the stream decoded by the QoS-Monitoring Probe(s) to this perceived QoS measurement tool and have a quantitative measure of the quality degradation. The necessary resources were not available at the time the work reported was carried out so the QoS analysis has been left at a qualitative level.

\section{ACKNOWLEDGMENT}

The authors wish to express special gratitude to all the partners collaborating in the IST Integrated Project INSTINCT (IPbased Networks, Services and Terminals for Converging systems) [17] for their valuable contributions to the research.

\section{REFERENCES}

[1] M. Lengdell, J. Pavon, M. Wakano, M. Chapman, and M. Kawanishi, "The TINA network resource model," Communications Magazine, IEEE, vol. 34, no. 3, pp. 74-79, Mar. 1996, DOI 10.1109/35.486806.

[2] A. Keller, "Managing the management: CORBA-based Instrumentation of Management Systems," in IFIP/IEEE International Symposium on Integrated Network Management (IM'99), Boston, MA, May 1999.

[3] F. Allamandri, J.-F. Le Boite, S. Campion, A. Centonza, A. Chernilov, J. Cosmas, E. Tsekleves, D. Garrec, M. Guiraudou, K. Krishnapillai, T. Levesque, M. Re, and L. Zheng, "Converged DVB-H and cellular telecommunications service architecture," in World Wireless Congress 2005, San Francisco, CA, May 26-28, 2005.

[4] A. Centonza, G. A. Taylor, T. Itagaki, T. Owens, J. Cosmas, and Y.-H. Song, "Stochastic user behaviour modelling and network simulation for resource management in cooperation with mobile telecommunications and broadcast networks," International Journal of Mobile Communications, vol. 4, no. 1, pp. 97-117, 2006.

[5] A. Centonza, P. Casagranda, T. Owens, J. Cosmas, and Y.-H. Song, "Management of Digital Video Broadcasting Services in Open Delivery Platforms," International Journal of Mobile Communications, vol. 5, no. 2, pp. 186-214, 2007

[6] J. Cosmas, L. Cruickshank, L. Elgohari, T. Itagaki, A. Lucas, K. Krishnapillai, and L. Zheng, "Rapid prototyping and fast user trial of multimedia broadcast and cellular services," International Journal of Mobile Communications, vol. 3, no. 3, pp. 263-286, 2005.

[7] Debian 2005 [Online]. Available: www.debian.org

[8] Distributed Management Task Force (DMTF), Inc. [Online]. Available: http://www.dmtf.org/home

[9] "DMI-to-SNMP Mapping Specification," Desktop Management Task Force (DMTF), Inc., Nov. 25, 2005, ver. 1.0.

[10] "Specification of the Representation of CIM in XML," Desktop Management Task Force (DMTF), Inc., Dec. 09, 2004, Preliminary Version 2.2.

[11] "Transmission System for Handheld Terminals (DVB-H)," Nov. 2004, ETSI EN 302 304, vol. 1.1.1.

[12] "Digital Video Broadcasting; Measurements Guidelines for DVB systems," Aug. 1997, ETSI TR 101290. 
[13] "Tractors and Machinery for Agriculture and Forestry-Serial Control and Communications Data Network-Part 5: Network Management," Nov. 2005, ISO 11783-5:2001.

[14] P. Jacobs and B. Davie, "Technical challenges in the delivery of interprovider QoS," Communication Magazine, IEEE, vol. 43, no. 6, pp. 112-118, 2005.

[15] "Simple Network Management Protocol (SNMP)," May 1990, RFC 1157.

[16] L. Schweizer, "Convergence: A challenge for mobile telecommunications operators-The case of the German T-Mobile," International Journal of Mobile Communications, vol. 4, no. 2, pp. 143-162, 2006.

[17] F. Allamandri, S. Campion, A. Centonza, A. Chernilov, J. P. Cosmas, A. Duffy, D. Garrec, M. Guiraudou, K. Krishnapillai, T. Levesque, B. Mazieres, R. Mies, T. Owens, M. Re, E. Tsekleves, and L. Zheng, "Service Platform for Converged Interactive Broadband Broadcast and Cellular Wireless," IEEE Trans. Broadcasting, vol. 53, no. 1, pp. 200-211, Mar. 2007.
[18] "Digital Video Broadcasting (DVB), Measurement Guidelines for DVB systems," 2001-5, ETSI TR 101290 v1.2.1.

[19] "Netem," Netem—Network Emulator [Online]. Available: http://developer.osdl.org/shemminger/netem/

[20] [Online]. Available: www.istintermon.org/

[21] [Online]. Available: www.ist-mome.org/

[22] [Online]. Available: www.istscampi.org/

[23] [Online]. Available: www.ist-lobster.org/

[24] [Online]. Available: www-st.inf.tu-dresden.de/Aquila/

[25] [Online]. Available: www.enthrone.org/

[26] M. Sidibe and A. Mehaoua, "QoS monitoring for end-to-end heterogeneous networks configurations management," in IEEE International Conference on Automation, Quality and Testing, Robotics 2008, vol. 1, pp. 364-368, 978-1-4244-2576-1. 